\title{
Virtual marathon runners hold world record
}

Dentist Shishir Yadav, of Bishopdown Farm Dental Practice in Salisbury and Smile Dental Care in Eastleigh, is proud to have been mentioned in the Guinness Book of World Records for taking part in the Virgin Money London Marathon (UK) on 4 October 2020 and therefore contributing to a record for the most people $(37,966)$ to run a remote marathon in 24 hours.

Keen sportsman and runner Dr Yadav (pictured) also ran the Virtual London Marathon this year, raising funds for Macmillan Cancer Support, and hopes to be lucky enough to run the actual marathon in the future.

Dr Yadav said: 'Keeping fit and having an active lifestyle can significantly reduce the chances of chronic health conditions, mental health disorders, and even premature death. I think placing importance on exercise as a dental team sets the perfect example for your patients. If your patients can see you keeping fit and healthy, they will see you as more than just their dentist, but a role model too'

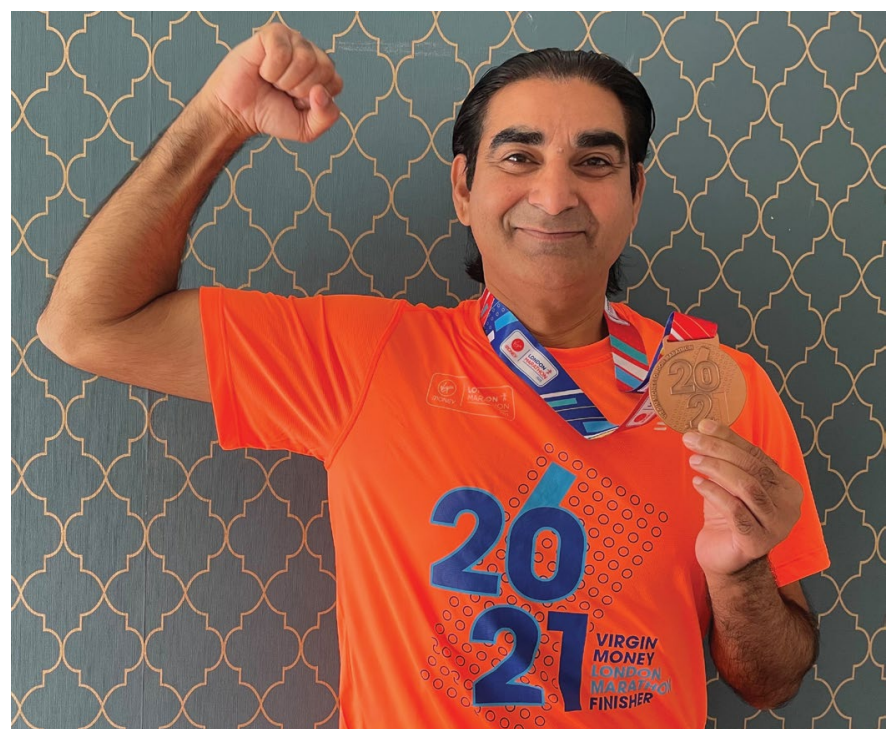

\section{British Orthodontic Conference 2021}

By William Timmis, Dental Foundation Trainee, Yorkshire and the Humber, UK

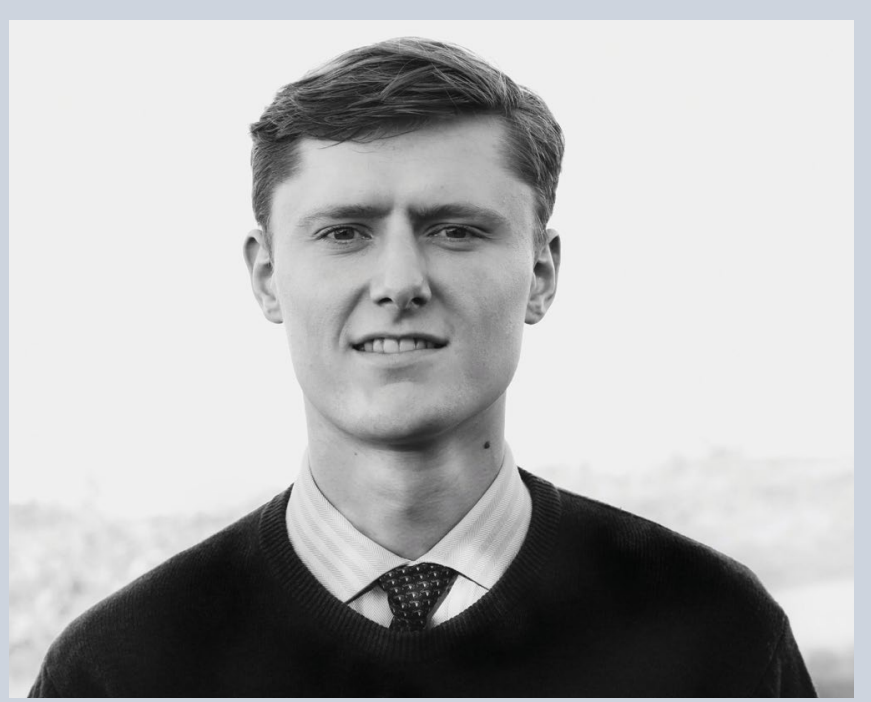

William Timmis

The annual British Orthodontic Conference (BOC) took place between 16-18 September 2021 at the Midland Hotel, Manchester. The British Orthodontic Society have faced an exceptionally busy 18-month period, having been forced to cancel and re-arrange the 2020 conference, due to COVID-19 restrictions. In order to prevent further postponement, the BOC 2021 was presented in a hybrid event format. An integrated approach was taken, combining in-person presentations and a trade exhibition with online virtual access. Despite diverging away from traditional BOC arrangements, it ensured the provision of networking opportunities between members and delegates, regardless of their location or access to the event. Whilst there were too many presentations to cover individually, a brief summary of the conference is outlined below.
The Pre-conference Course (15 September) offered a hands-on seminar, followed by small, group case-based discussions. This primarily focused on establishing an enhanced appreciation of joint restorative and orthodontic approaches in diagnosing and managing complex patient cases. The interface between both approaches demonstrated methods to tackle cases such as hypodontia.

Day one of the main scientific programme included the Chapman Prize 2020 presentation. This was delivered jointly by Professor Padhraig Fleming and Dr Pratik Sharma, where they presented their mixed-methods research study. It incorporated a randomised control trial (RCT) investigating the effectiveness of full- versus part-time Twin Blocks appliance wear, whilst a qualitative evaluation was utilised to explore the factors affecting compliance with prescribed wear protocols. This was later followed by Dr Simon Littlewood's presentation on enhancing the clinical application of evidence-based tooth retention regimes. It was supported by contemporary research into orthodontic-bonded retainers, ultimately helping mitigate the risk of relapse.

Dr Sarah Good delivered another interesting lecture: 'Things I learned from hanging out with kids'. The presentation underlined the significance of providing closely aligned, joint paediatricorthodontic services and incorporated the presiding evidence that guides the management of complex cases including molar incisor hypomineralisation.

The Northcroft Memorial Lecture 2020 was delivered by Professor Jonathan Sandler on the second day of the BOC 2021. A breadth of material was covered, ranging from the importance of serial clinical photography, to osseointegrated palatal implants as substitutes for headgear, and the effective incorporation of temporary anchorage devices (TADs) into orthodontic practice, to name just a few. RCTs were provided as supplementary, supportive material 


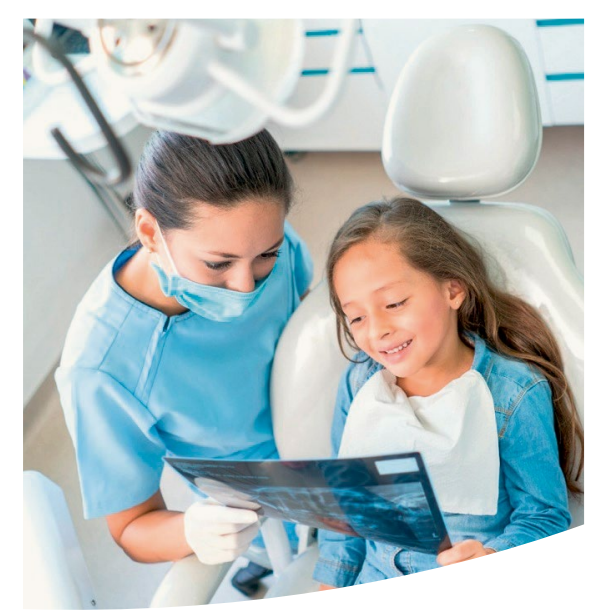

Combined online child protection and adult safeguarding

\section{courses}

An introduction to child protection/ adult safeguarding

A general combined course for all dental staff who come into contact with children and vulnerable adults.

\author{
3 hrs. verifiable CPD $\begin{aligned} & \text { Development } \\ & \text { outcomes }\end{aligned}$ (A) (B) (C) (D) \\ In line with Level 1
}

Further training in child protection/ adult safeguarding

A combined course developed for the nominated safeguarding lead and deputy within a dental practice, who have already completed introduction training

\section{3 hrs. verifiable CPD Development (A) (B) (C) (D) In line with Level 2 \\ These online training courses are delivered by The Child Protection Company and fulfil the safeguarding outcomes as specific by CQC/ROIA HWB and fulfil the safeguarding Outcomes specific by CQC/RQIA/HWB.}

Cost: $£ 27.90$

BDA Expert members: Free

Essential and Extra

BDA members:

$10 \%$ discount

Discount applies to all when the member books!

\section{bda.org/safeguarding}

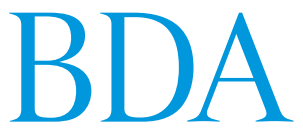

British Dental Association

The BDA is owned and run by its members.
1 throughout and provided a comprehensive, evidence-based overview of the techniques discussed. This was later followed by 'The art of living when you know you're going to die', delivered by Dr Phil Hammond, Private Eye's MD. The presentation was amusing, yet equally thought-provoking, as it drew upon Dr Hammond's experience in the NHS, the impact of COVID-19 and the overall importance of good dietary practices. Day two offered a holistic overview of specific clinical practices, merged with in-depth, personal experiences.

Dr Aliki Tsichlaki presented the Chapman Prize 2021 lecture on the final day. Her winning PhD research looked into the development of a standardised, core orthodontic outcome set for future implementation across clinical trials. The standardised measures installed would look to enable improved outcome consistency between future orthodontic studies and enhanced research yield, while guaranteeing an improved measurement of outcomes significant to all stakeholders including clinicians and patients. Dr Nicky Mandall followed on from this later, reflecting upon her multi-centre RCT. It assessed both the clinical and psychosocial influence of Twin Block treatment delay, as well as the associated dentofacial changes. Dr Mandall continued by discussing how the specific research has since changed her clinical practice, as well as future proposals for a further RCT.

The success of the BOC's 2019 dual parallel track sessions, known as 'Driving Private Practice' and 'So, you want to know about orthodontics', confirmed their repeated presence in the 2021 programme schedule. The latter was particularly beneficial for those interested in gaining an increased appreciation of the orthodontic speciality training pathway. It was key in highlighting the associative clinical and research commitments required. Dr Guy Deeming's presentation meanwhile discussed a range of key factors necessary in contributing towards a more cohesive dental team and its unified benefit in enhancing practice performance. A full two-day programme was additionally offered for dental care professionals, aimed at providing a range of informative sessions, spanning from medical emergencies to mindfulness.

OrthoTED sessions also returned for a consecutive year. With an overarching aim at spreading awareness around 'Orthodontic Technology, Education and Design', the 2021 OrthoTED lecture series offered a dynamic and informative insight into various thought-provoking concepts. Topics ranged from the provision of oral health advice through social media, digital marketing and alternative team leadership approaches. This was supported by Clinical Pearls, providing more specific clinical advice around the utilisation of floss ligatures, video consultations, efficacious pontic-archwire adhesion and much more. This was a highly informative series of lectures, inspiring the future of dentistry.

In summary, BOC 2021 facilitated a widely interactive learning opportunity, addressing various dental techniques and practices. I would advise all of those with an interest in orthodontics to attend the BOC 2022 in Birmingham. Finally, I would like to extend my thanks to Dr David Waring, the BOC 2021 Chair and the rest of the BOC committee for organising such a diverse, engaging and accessible event. 